Research Paper

\title{
The role of $\beta$-catenin in the initiation and metastasis of TA2 mice spontaneous breast cancer
}

Dan Zhang1\#, Fei Fei2\#, Shuyuan Li#, Yongjie Zhao ${ }^{4}$, Zhengduo Yang ${ }^{1}$, Jie Qu², Xipeng Zhang 3 , Yu Yin ${ }^{5}$, Shiwu Zhang ${ }^{\boxplus}$

1. Department of Pathology, Tianjin Union Medical Center, Tianjin, 300121, China

2. Nankai University School of Medicine, Nankai University, Tianjin, 300071, China

3. Department of Colorectal Surgery, Tianjin Union Medical Center, Tianjin, 300121, China

4. Department of General Surgery, Tianjin Union Medical Center, Tianjin 300121, China

5. Department of Pathology, Anhui Medical University, Hefei 230032, China

\#These authors equally contributed to the paper.

$\triangle$ Corresponding author: Shiwu Zhang, Department of Pathology, Tianjin Union Medical Center, Tianjin 300121, China. Tel.: (86)-13652136865. E-mail: shiwu7211@126.com.

(C) Ivyspring International Publisher. This is an open access article distributed under the terms of the Creative Commons Attribution (CC BY-NC) license (https://creativecommons.org/licenses/by-nc/4.0/). See http://ivyspring.com/terms for full terms and conditions.

Received: 2017.02.19; Accepted: 2017.04.10; Published: 2017.07.05

\begin{abstract}
Purpose: Tientsin Albino 2 (TA2) mice have a high incidence of spontaneous breast cancer. Tumor initiation is related to mouse mammary tumor virus (MMTV) infection. MMTV is hormonally regulated and may promote tumor formation via $W n t / \beta$-catenin signaling pathway. This study attempts to clarify the relationship between $\beta$-catenin expression and the initiation and metastasis of spontaneous breast cancer in TA2 mice.

Materials and Methods: Pathological samples illustrating the development of spontaneous breast cancer in TA2 mice were collected and the presence of virus particles was verified in the cancer tissue by electron microscope. Expression of $\mathrm{Wnt} / \beta$-catenin signaling-pathway-related proteins including

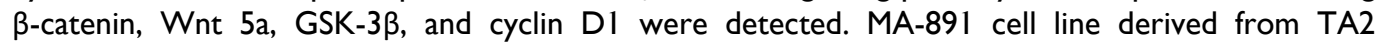
spontaneous breast cancer was cultured and siRNA was used to inhibit the expression of $\beta$-catenin in the primary culture cell line. Cell cycle analyses and comparisons of the invasiveness and migration capability of tumor cells were performed before and after $\beta$-catenin inhibition. Downstream protein expression of $\beta$-catenin was studied by western blot, co-immunoprecipitation assay. Tumorigenesis and metastasis were compared with that of negative control, siRNA control, and siRNA $\beta$-catenin- 1512 . Furthermore, proteins related to the proliferation and invasion of tumor were detected by western blot.

Results: $\beta$-catenin expression was found to be located in the membrane and cytoplasm in normal mammary tissue and precancerous lesions, respectively. However, in the breast cancer tissue, $\beta$-catenin expression was located in the nuclei. After transfection with siRNA-1512, the cells showed decreased proliferation, invasiveness and migration capability, tumorigenicity, and metastasis, and the expression of the proteins related to tumor proliferation and metastasis such as c-myc, Cyclin DI, MMP-9, and VEGF were down-regulated.
\end{abstract}

Conclusion: These results confirmed that the expression and location of $\beta$-catenin were associated with the initiation and metastasis of spontaneous breast cancer in TA2 mice.

Key words: Tientsin Albino 2; $\beta$-catenin; MA-891; Breast cancer.

\section{Introduction}

Breast cancer is a very common form of human cancer and has become a serious threat to women's health. Surgery accompanied with radiotherapy and chemotherapy is the most successful treatment strategy for breast cancer. However, $40 \%$ of patients die of advanced breast cancer recurrence and 
metastasis [1]. The initiation of breast cancer is hormone-dependent. The mechanisms of hormonally regulated tumorigenesis in breast cancer are complicated and involve different signaling pathways [2-5].

Mice are frequently used in genetic studies. TA2 mice model is one of the spontaneous breast cancer (SBC) models established by Tianjin Medical University, after several decades years of selection. The incidence of SBC in TA2 mice is greater than $80 \%$ in the absence of any external chemical stimuli. In our previous study, we proved that the high incidence of TA2 SBC had two explanations: 1) Gravidity and the frequency of pregnancy and 2) Presence of mouse mammary tumor virus (MMTV) [6, 7]. Our results were supported by reports on the successful amplification of an entire proviral structure with strong homology to MMTV from human breast cancer DNA. MMTV sequences contain hormone-responsive elements (HRE), transcription enhancer factor-1 (TEF-1) family elements, and an open reading frame (ORF) for superantigen (SAg). Additionally, animal studies may help us to understand breast tumorigenesis in humans. Our previous studies have confirmed that combined exogenous estradiol and progesterone treatment induce the initiation of breast cancer in TA2 mice without ovaries by the increase in expression of Wnt1, Wnt5a, Wnt5b, and Wnt $10 \mathrm{~b}$ mRNAs in their mammary tissues. Nevertheless, the Wnt/ $\beta$-catenin signaling pathway is involved in tumor initiation and is associated with malignant behaviors in many kinds of tumors $[8,9]$.

In the presence of Wnt proteins, the cytoplasmic protein dishelleved (Dsh) inhibits the activity of glycogen synthase kinase $3 \beta$ (GSK-3 $\beta$ ). Accumulation of $\beta$-catenin can translocate to the nucleus and act as a transcriptional regulator. Nuclear $\beta$-catenin then associates with the lymphoid enhancer factor/T-cellspecific transcription factor (LEF/TCF) family and activates transcription of Wnt target genes, such as c-myc and Cyclin D1 [10-12]. As a key regulator in the Wnt signaling pathway, $\beta$-catenin plays an important role in the regulation of mammary development and tumorigenesis. Depending on its cell localization, $\beta$-catenin is known to perform different functions. Studies in mice suggest that dysregulated $\beta$-catenin signaling increases breast cancer risk by inducing stem and early progenitor cell accumulation [13, 14]. Meanwhile, increased cytoplasmic and nuclear $\beta$-catenin levels have been documented in $40 \%$ of primary human breast cancers and have been correlated with poor prognosis and patient survival [15-19].

In this study, we collected different pathologic samples during the process of TA2 SBC development and observed the location of $\beta$-catenin expression.
Furthermore, MA-891cell line, derived from TA2 mice with SBC, was used as a model to investigate the underlying molecular mechanism responsible for TA2 SBC development and metastasis.

\section{Materials and methods}

\section{TA2 mice with SBC}

Eleven female TA2 mice and three male TA2 mice were raised for more than 14 months. Nine TA2 mice had SBC. Two of these mice had bred two times, one mouse had bred three times, three mice had bred four times, one mouse each had bred five, six, and seven times. Thirteen tumor masses were harvested after killing the mice. A part of the tumor without necrosis that was obtained from each mouse was frozen. Part of tumor and the "normal mammary gland" from the mice with SBC were fixed with formalin and made into paraffin-embedded blocks. The other details of TA2 SBC were as listed in the supplementary table 1. The Animal Ethics Committee of National Research Institute approved the animal experimentation protocols and all animal experiments were performed according to guidelines (Guidelines for the Care and Use of Laboratory Animals) established by the Chinese Council on Animal Care.

Paraffin-embedded tissue included normal breast mammary gland, ductal hyperplasia and atypical ductal hyperplasia of mammary gland tissue from TA2 SBC, intraductal carcinoma, invasive carcinoma, and the metastatic foci of lung which were collected to observe the pathologic process of TA2 SBC development. These with paraffin-embedded blocks were sectioned for immunohistochemical (IHC) staining.

\section{Electron microscopy}

Electron microscopy was performed by a standard procedure. Specimens from TA2 SBC were fixed with glutaraldehyde. Ultra-thin sections were examined to observe the virus particles using a JEM-1010 electron microscope.

\section{Cell line of TA2 SBC}

The MA-891 cell line was obtained from Biohermes, Inc. (Shanghai, China) and maintained in RPMI 1640 medium (GIBCO, NY, USA) containing $10 \%$ heat-inactivated fetal bovine serum (ExCell Biology, USA), penicillin (100 units/mL), and streptomycin $(100 \mu \mathrm{g} / \mathrm{mL})$ in a $5 \% \mathrm{CO}_{2}$ incubator at $37^{\circ} \mathrm{C}$.

\section{Transient siRNA transfection}

$\beta$-Catenin and the negative control siRNA oligonucleotides were synthesized by Gene-pharma (Shanghai, China). The MA-891 cells were grown and 
transfected with either $\beta$-catenin or the negative control siRNA oligonucleotides using Lipofectamine 2000 (Invitrogen, Carlsbad, CA, USA) according to the manufacturer's instructions. The sequences of siRNA oligonucleotides were as listed in supplementary table 2.

\section{Quantitative reverse transcriptase PCR}

Total RNA was isolated from cells using the TRizol (Invitrogen, USA) and reversely transcribed into cDNA according to the manufacturer's instruction. Aliquots of these cDNA samples were used for qPCR with an SYBR Premix DimerEraserTM (TOYOBO, Japan). Thermal cycling was performed on DNA Engine Peltier Thermal cycler (Bio-Rad, Hercules, CA, USA) for RT and ABI 7300 HT Sequence Detection System (Applied Biosystems, USA) for qPCR. Expression levels of $\beta$-catenin were normalized to GAPDH levels. The primer sequences for $\beta$-catenin were $5^{\prime}$-TGGAGCCGGACAGAAAAGC$3^{\prime}$ and 5'-CTTGCCACTCAGGGAAGGA-3'. The primer sequences for GAPDH were 5'-TATGTCGTGGA GTCTACTGGT-3' and 5'-GAGTTGTCATATTTCTCG TGG-3'.

\section{Immunofluorescence staining}

Transiently transfected MA-891 cells were cultured on cover slips. The cells were washed with $1 \times$ phosphate buffered saline (PBS) and fixed with $4 \%$ paraformaldehyde for $1 \mathrm{~h}$ at room temperature. After being washed with PBS, the cover slips were incubated with $1 \times$ PBS containing $5 \%$ bovine serum albumin and $1 \%$ Triton $\mathrm{X}-100$ for $15 \mathrm{~min}$ at room temperature and then incubated with primary antibodies against $\beta$-catenin (Abcam, USA, dilution: 1:600) at room temperature for $2 \mathrm{~h}$. They were then washed with $1 \times$ PBS for 30 min to remove nonspecific binding, followed by reaction with the secondary antibody, and then a goat anti-mouse immunoglobulin G (IgG)-Texas Red (Invitrogen, USA) was added to the slides. The slides were incubated for $1 \mathrm{~h}$ at the room temperature. The nuclei were visualized with DAPI staining. Cell morphology was visualized and photographed with fluorescence microscopy.

\section{Cell cycle analysis}

Cell cycle analysis was performed with flow cytometry (FCM). MA-891 cells with different treatments were harvested, washed in PBS, and fixed at $4{ }^{\circ} \mathrm{C}$ overnight with $70 \%$ ethanol. Cells were washed with cold PBS and incubated at $4{ }^{\circ} \mathrm{C}$ for 30 min with PI/RNase solution (Becton Dickinson, San Jose, CA). Samples were analyzed with a FACScan flow cytometer (Becton Dickinson, San Jose, CA) equipped with a $488 \mathrm{~nm}$ laser.

\section{Cell viability MTT assay}

Ten $100 \mu \mathrm{l}$ aliquots containing $5 \times 10^{3}$ MA-891 cells in logarithmic growth phase from each treatment were prepared and seeded into 96 well plates. The cells were incubated for $72 \mathrm{~h}$, and then $20 \mu \mathrm{l}$ of MTT (3-[4,5-dimethylthiazol-2-yl]-2,5 diphenyl tetrazolium bromide reagent (Amresco 0793, USA) was added to each well, including three control wells containing medium alone which were used as blanks for absorbance readings. After $4 \mathrm{~h}$ of incubation, $100 \mu \mathrm{l}$ of detergent reagent was added to all wells after the purple precipitate was clearly visible. The absorbance in each well was measured at $490 \mathrm{~nm}$. The optical density (OD) value was calculated as the average value of ten readings after the subtraction of the average value of the blank.

\section{Tumor cell migration and invasion assay}

Invasiveness was determined using cell culture inserts for 24-well plates with the bottom sealed by an $8-\mu \mathrm{m}$ pore polycarbonate-filter coated with $50 \mu \mathrm{g} / \mathrm{L}$ of liquid Matrigel (Chemicon International, Inc.) in a 1:8 dilution. MA-891 cells were cultured in RPMI 1640 without sera at $37^{\circ} \mathrm{C}$ with $5 \% \mathrm{CO}_{2}$ and were adjusted to $1 \times 10^{5}$ cells $/ \mathrm{mL}$. Hundred microliters of serum-free RPMI containing $1 \times 10^{4}$ cells were seeded into the inserts. The lower chambers were filled with $500 \mu \mathrm{l}$ RPMI plus $10 \%$ FBS. Chambers were incubated at $37^{\circ} \mathrm{C}$ in a $5 \% \mathrm{CO}_{2}$ atmosphere for $24 \mathrm{~h}$. Cells on the upper surface of the filter were removed using a cotton swab. Cells that invaded through the coated filter to the lower surface were then stained with $0.1 \%$ crystal violet for photography. Data are expressed as means \pm standard error, $n=4$. For migration experiments, Transwell chambers were coated with fibronectin on the upper surface of the filter, and then $1 \times 10^{4}$ MA-891 cells in $100 \mu \mathrm{l}$ RPMI medium with $10 \%$

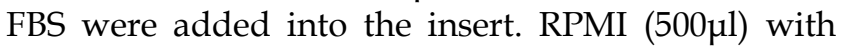
$10 \%$ FBS was added into the lower chambers. After incubation at $37^{\circ} \mathrm{C}$ for $24 \mathrm{~h}$, cells on the upper surface of the filters and the fibronectin were removed using a cotton swab. Crystal violet $(0.1 \%, 500 \mu l)$ was added into the 24-well plate for $30 \mathrm{~min}$ at $37^{\circ} \mathrm{C}$ and cells that invaded through the coated filter to the lower surface were stained. Data are expressed as means \pm standard error, $n=4$.

\section{Co-immunoprecipitation (Co-IP) assay}

MA-891 cells were harvested. Extracts were incubated overnight with $1 \mu \mathrm{g}$ each of anti-TCF-4 (Santa Cruz, Heidelberg, Germany) in the presence of Protein G beads, a component of the Protein G Immunoprecipitation kit (Sigma, St. Louis, MO, USA). Extracts that were incubated without anti- $\beta$-catenin was used as the control. Resulting complexes were 
washed, denatured, and eluted according to the manufacturer's instructions. Western blotting was performed using the ECL procedure with anti- $\beta$-catenin antibody (Santa Cruz, USA, 1:500).

\section{Western blot}

MA-891 cells and tumor tissues derived from MA-891 xenografts were harvested and lysed with a radioimmunoprecipitation assay (RIPA) buffer supplemented with proteinase inhibitor cocktail. The protein concentrations were determined and the proteins were separated on a $10 \%$ SDS-polyacrylamide gel and transferred to nitrocellulose membranes. The membranes were incubated with antibodies against $\beta$-catenin (Santa Cruz, USA), c-myc (Boster, Wuhan, China), Cyclin D1 (BD Biosciences, Sparks, MD), and matrix metalloproteinase 9 (MMP-9, Santa Cruz, CA, USA), Vascular endothelial growth factor (VEGF) (Boster, Wuhan, China), or $\beta$-actin (Sigma). The protein bands were detected by incubating with horseradish peroxidase (HRP)-conjugated antibodies and visualized using the Super Signal West Pico Chemiluminescent Substrate Kit (Thermo Scientific, Rockford, IL).

\section{IHC staining}

Four-micrometer sections of formalin-fixed, paraffin embedded engrafted animal tumor tissues were mounted on poly L-lysine-coated slides. Slides were deparaffinized in xylene, and rehydrated and washed with PBS before subjecting it to antigen retrieval. Endogenous peroxidase activity was blocked with 3\% hydrogen peroxide solution in $50 \%$ methanol for $10 \mathrm{~min}$ at room temperature. After the nonspecific protein binding and the endogenous peroxidase activity were blocked, the sections were incubated with a rabbit polyclonal anti- $\beta$-catenin (Abcam, USA, dilution: 1:600), Wnt 5a (anti-rabbit, Beijing Bioscience Biotechnology Co. Ltd. Beijing, China; 1:100 dilution), GSK-3 $\beta$ (anti-rabbit, Beijing Bioscience Biotechnology Co. Ltd. Beijing, China; 1:100 dilution), and Cyclin D1 (ZA-0101, anti-rabbit, Beijing Zhongshan Biotechnology Co. Ltd. Beijing, China; 1:100 dilution) overnight at $4{ }^{\circ} \mathrm{C}$. After reaction with MaxVision (Maixing, Fujian, China) for 20 min, the signal was detected in the presence of the chromogen 3,3'-diaminobenzidine (Maixing Fujian, China). After counterstaining the nuclei with hematoxylin, the slides were dehydrated and mounted with coverslips.

\section{Animal xenograft assay}

Eighteen female TA2 mice (6-week-old) were obtained from the animal center of Tianjin Medical University and divided into three groups: negative control, siRNA control, and siRNA $\beta$-catenin-1512. Each group contained six mice. MA-891 cells were washed twice in ice-cold serum-free RPMI 1640 medium, counted for viable cells, and resuspended in serum-free RPMI 1640 medium. 1×106 MA-891 cells were injected in the right flank of each mouse in a volume of $0.1 \mathrm{~mL}$. Starting on the $7 \mathrm{th}$ day after injection, tumors were measured every two days. The tumor size was calculated according to the following formula: Tumor volume $\left(\mathrm{cm}^{3}\right)=\left(\right.$ length $\times$ width $\left.{ }^{2}\right) / 2$. A tumor growth curve was plotted based on tumor size. On the 19th day after inoculation, all mice were sacrificed, and the tumor tissues were harvested and weighed. The mouse liver and lung were removed intact to detect any metastatic tumors. This study was approved by the Institutional Animal Care and Use Committee of Tianjin Union Medicine Center (IACUC).

\section{Statistical analysis}

Statistical software SPSS 13.0 was used to evaluate the data and $P<0.05$ was defined as statistically significant. Differences in cell proliferation, invasiveness, and migration among negative controls, siRNA control, and siRNA $\beta$-catenin-1512 were assessed using the ANOVA test. Mann-Whitney Test was performed to compare differences in tumor weight among the three groups.

\section{Results}

\section{Morphological changes during the process of TA2 SBC development}

SBC appeared in nine of eleven female TA2 mice with different frequencies of pregnancy; the average time taken by the mice from birth to the occurrence of SBC was about 285.44 days. Lung metastasis was detected in four mice with SBC and liver metastasis appeared in two mice with SBC, whereas, both lung and liver metastasis was detected in one mouse; the other two mice had no metastasis of lung or liver. Two tumor masses were found in four mice.

Breast tissue and different lesions for TA2 SBC development were used for $\mathrm{H} \& \mathrm{E}$ and IHC staining. In the normal breast mammary gland from TA2 mice without pregnancy, the mammary ductal structure was integrated and regularly shaped, and the epithelial cells appeared normal (Fig. 1A -a). However, the mammary gland tissue from TA2 mice with SBC showed ductal hyperplasia (Fig. 1A, b). Different morphological changes during the process of TA2 SBC development including the atypical ductal hyperplasia (Fig. 1A, c), intraductal carcinoma (Fig. 1A, d and e), invasive breast carcinoma (Fig. 1A, f), and lung (Fig. 1A, g) and liver (Fig. 1A, h) metastatic foci were observed. 

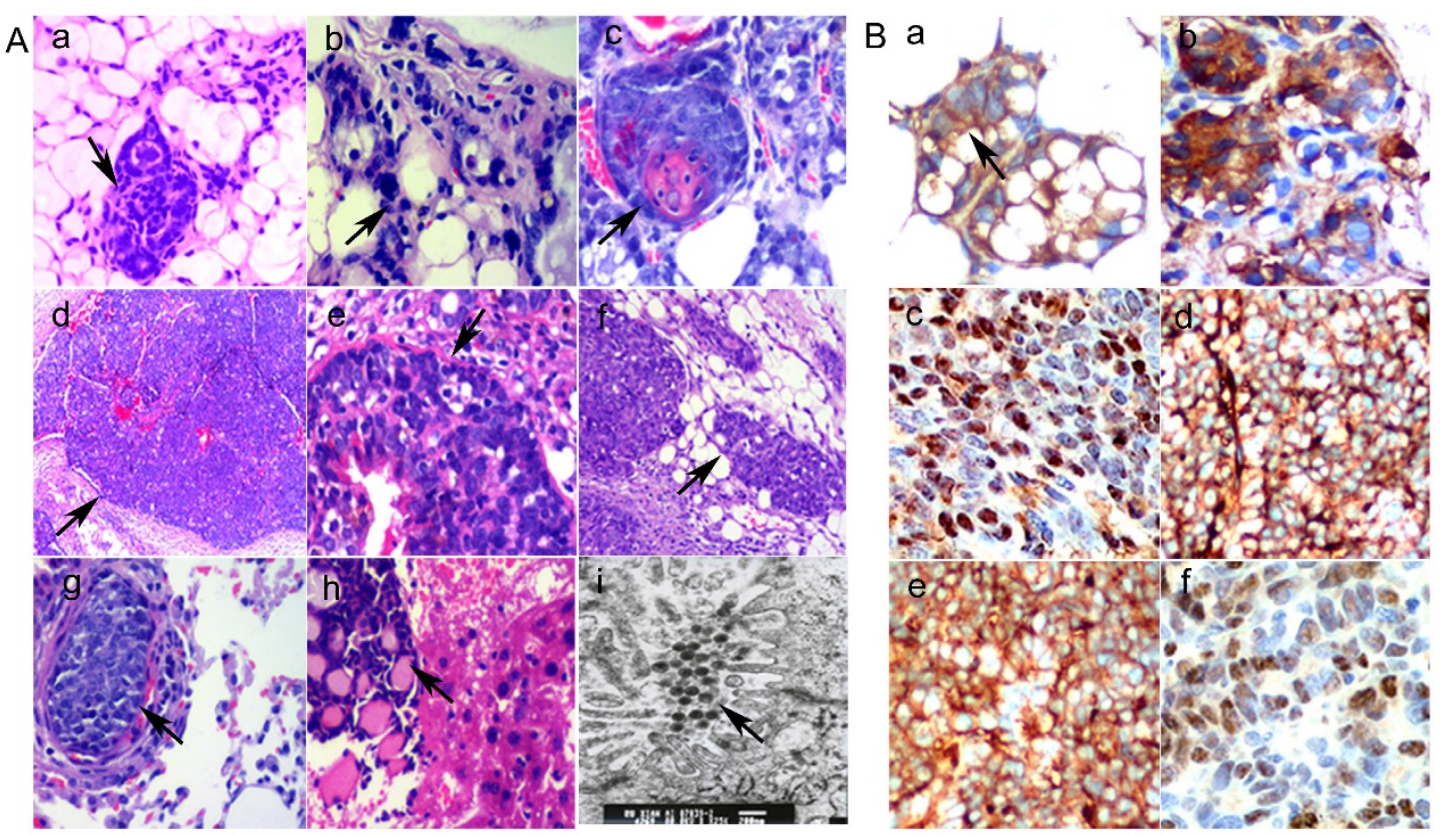

Figure 1. A. Morphological changes in different breast lesions in TA2 mice. a) Normal breast mammary gland tissue from TA2 mice without SBC ( $\times 100$, black arrowheads). b) Ductal hyperplasia of breast mammary gland tissue from TA2 mice with SBC ( $\times 200$, black arrowheads). c) Atypical ductal hyperplasia of breast mammary gland tissue from TA2 mice with SBC $(\times 200$, black arrowheads). d) Spontaneous intraductal carcinoma of TA2 mice $(\times 100$, black arrowheads). e) Spontaneous intraductal carcinoma of TA2 mice, the black arrow points at the intact basement membrane $(\times 200$, black arrowheads). f Spontaneous invasive breast carcinoma of TA2 mice ( $\times 100$, black arrowheads). $g$ ) The metastatic foci of lung in TA2 mice with SBC ( $\times 200$, black arrowheads). $h)$ The metastatic foci of liver in TA2 mice with SBC $(\times 100$, black arrowheads). i) This electron micrograph of SBC from TA2 mice showing the virus particles $(\times 3000$, black arrowheads). B. Expression of $\mathrm{Wnt} / \beta$-catenin signaling pathway proteins. a) $\beta$-catenin expression in normal mammary tissue and the membranes of mammary gland epithelium were positive for $\beta$-catenin $\mathrm{IHC}$ staining (IHC, $\times 200$, black arrowheads). b) $\beta$-catenin expression in precancerous lesion in TA2 mice and the cytoplasm of mammary gland epithelium were positive for $\beta$-catenin IHC staining (IHC, $\times 200)$. c) $\beta$-catenin expression of SBC tissue in TA2 mice and the nuclei of mammary gland epithelium were positive for $\beta$-catenin IHC staining $(\mathrm{IHC}, \times 200)$. d) Wnt 5 a expression was located in the cytoplasm of SBC in TA2 mice $(\mathrm{IHC}, \times 200)$. e) GSK-3 $\beta$ expression was located in the cytoplasm of SBC in TA2 mice (IHC, $\times 200)$. f) Cyclin D1 expression was located in the nuclei of SBC in TA2 mice (IHC, $\times 200)$

\section{Results from electron microscopy}

Our previously published paper [7] confirmed that the presence of MMTV was related to the initiation of TA2 SBC. MMTV is hormonally regulated and indirectly promotes tumor formation by causing activation of Wnt oncogenes through insertional mutagenesis. Results of electron micrograph of TA2 SBC tissue showed that there were many virus particles in the cytoplasm of cancer cells (Fig. 1A, i).

\section{Expression and localization of the Wnt/ $\beta$-catenin signaling pathway-related proteins in TA2 SBC tissues}

In order to confirm the association of the Wnt/ $\beta$-catenin signaling pathway in TA2 SBC, localized expressions of $\beta$-catenin in normal mammary gland tissue, precancerous lesion of mammary gland (mammary gland from TA2 mice with SBC), and TA2 SBC was determined by IHC staining. $\beta$-Catenin was only expressed in the membrane of normal mammary gland epithelial cells of TA2 mice (Fig.1B, a). In precancerous lesion of mammary gland in TA2 SBC, the expression of $\beta$-catenin expression was located in the cytoplasm (Fig.1B, b). Both the cytoplasm and nucleus of TA2 SBC cells displayed expression of $\beta$-catenin (Fig.1B, c). Furthermore, other proteins including Wnt 5a, GSK$3 \beta$, and cyclin D1 were detected; Wnt 5a (Fig. 1B, d) and GSK-3 $\beta$ (Fig. 1B, e) expression were located in the cytoplasm of TA2 SBC, whereas, Cyclin D1 expression was located in the nuclei of TA2 SBC (Fig. 1B, f).

\section{Knockdown of $\beta$-catenin expression using siRNA in MA-891 cells}

We designed and synthesized four different siRNAs: $\beta$-catenin-459, $\beta$-catenin-777, $\beta$-catenin-1512, and $\beta$-catenin-2254. After transfection, MA-891 cells were harvested. Results of real-time PCR (supplementary figure 1A), western blotting (supplementary figure 1B), and immunofluorescence microscopy (supplementary figure 1C) showed that the $\beta$-catenin expression levels in the four siRNA $\beta$-catenin groups, especially that of the siRNA $\beta$-catenin-1512 group, had decreased in comparison with that in the negative control group and siRNA control group. Accordingly, siRNA $\beta$-catenin-1512 group was chosen for the study. 
A

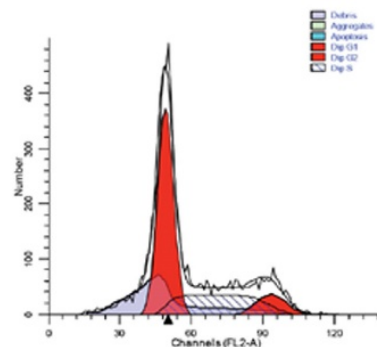

Negative control

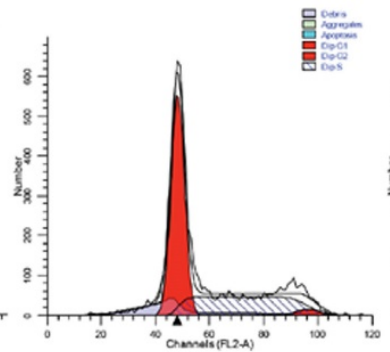

SiRNA control

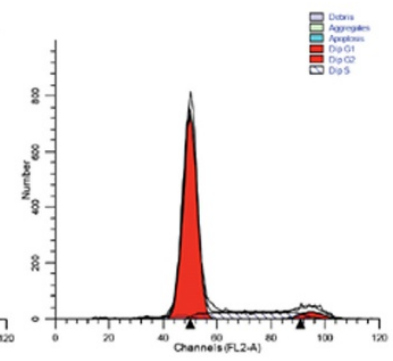

siRNA $\beta$-catenin-1512
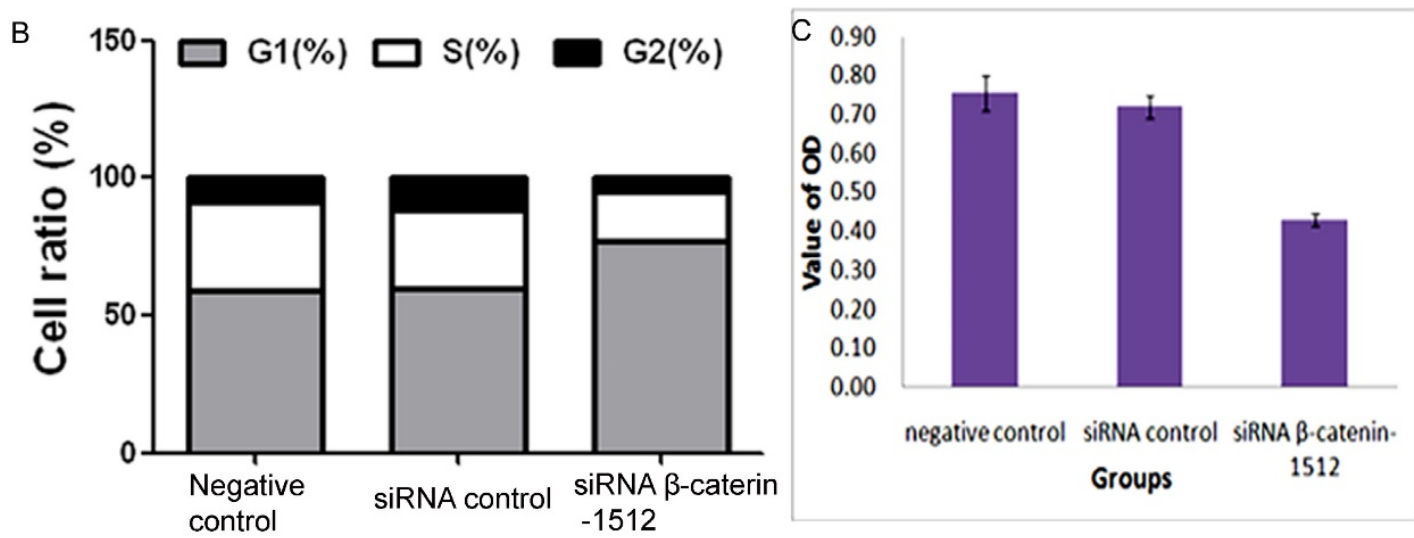

Figure 2. Different expression level of $\beta$-catenin regulated the cell cycle progression in MA-89 1 cells. A. Effects of the negative control, siRNA control and siRNA $\beta$-catenin-1512 treatments on the cell cycle of MA891 were analyzed by FCM. B. The ratios of MA-891 cells G1, S and G2 stages of cell division were analyzed by FCM. C. The proliferation of MA-891 cells in different groups was assessed by MTT. Proliferative potential of MA-891 cells with the negative control, siRNA control and siRNA $\beta$-catenin- 1512 treatments at $72 \mathrm{~h}$ after plating was determined using a modified MTT assay with $5 \times 104 / \mathrm{mL}$ cells. MA-891 cells treated with siRNA $\beta$-catenin-1512 exhibit a decrease in growth in comparison with the negative control and siRNA blank control.

\section{Knockdown of $\beta$-catenin expression blocked cell cycle progression in MA-89 1 cells}

Cell cycle analysis was performed via PI staining and FCM. The FCM analysis revealed that most of cells in the siRNA $\beta$-catenin-1512 group were arrested in the $\mathrm{G}_{1}$ phase of the cell cycle $(77.2 \pm 1.7 \%)$ compared to the negative control $(59.1 \pm 0.6 \%)$ and the siRNA control $(59.8 \pm 2.0 \%)$, and were found to decrease in both $S$ phase $(17.5 .1 \pm 0.3 \%)$ and $G_{2}$ phase $(5.3 \pm 1.5 \%)$ compared to the negative control group (32.1 $\pm 5.3 \%$ and $8.8 \pm 4.8 \%$ for $S$ and $G_{2}$ phases, respectively) and the siRNA control group $(28.6 \pm 3.5 \%$ and $11.5 \pm 1.6 \%$ for $S$ and $G_{2}$ phases, respectively) (Fig. $2 A$ and $2 B$ ). These results suggested that decreased $\beta$-catenin was related to the cell cycle of MA-891 cells.

\section{Knockdown of $\beta$-catenin expression decreased tumor cell proliferation, migration and invasiveness}

MA-891 cells treated with siRNA $\beta$-catenin-1512 exhibited a decreased proliferation rate, invasiveness, and migratory potential than the MA-891 negative control and the MA-891 siRNA control. We compared the proliferative potential among the negative control, the siRNA control, and the siRNA $\beta$-catenin-1512 group using the MTT assay. The MTT assay showed a decreased growth rate for the MA-891 siRNA $\beta$-catenin-1512 group compared to the negative control and the siRNA control $(\mathrm{F}=20.481, P=0.000$, Fig. 2C). Transwell assays were used to compare the invasiveness and migratory ability of MA-891 cells with that of the negative control, siRNA control, and siRNA $\beta$-catenin-1512. At $24 \mathrm{~h}$, the mean number of MA-891 cells that invaded across the filter was $136.0 \pm$ 4.97 for the negative control, $130.5 \pm 4.12$ for the siRNA control, and $55.50 \pm 4.43$ for the siRNA $\beta$-catenin-1512 group (Fig. 3A and 3B). Migratory cells of the negative control, siRNA control and siRNA $\beta$-catenin-1512 numbered $150.75 \pm 5.38,137.75 \pm 5.32$, and $64.0 \pm 5.88$, respectively (Fig $3 \mathrm{C}$ and $3 \mathrm{D}$ ). Results of the Transwell assay indicated that siRNA $\beta$-catenin-1512 inhibits the invasiveness and migration of MA-891 cells. There were statistical significances for cell invasiveness $(F=395.723$, $P=0.000)$ and migration $(F=286.032, P=0.000)$ among the negative control, the siRNA control, and the siRNA $\beta$-catenin-1512 groups.

\section{Knockdown of $\beta$-catenin expression downregulated expression of c-myc, cyclin DI, MMP-9 and VEGF proteins}

Further, to study the molecular mechanism of siRNA $\beta$-catenin-1512 in the inhibition of tumor 
proliferation, invasiveness, and migration, MA-891 cells with different treatments, western blot analysis was performed. Results showed that siRNA $\beta$-catenin -1512 inhibits the expression c-myc, cyclin D1, MMP-9, and VEGF (Fig. 3E). At the same time, the results of western blot after co-IP suggested that there was coexpression of $\beta$-catenin and TCF- 4 proteins (Fig. 3F).

\section{8. $\beta$-catenin expression in TA2 SBC tissues promoted tumor growth and metastasis}

MA-891 cell line derived from TA2 SBC had the ability to form tumor in TA2 mice. MA-891 cells with different $\beta$-catenin expression levels were injected in the TA2 mice and formed tumor. We detected the expression of $\beta$-catenin in normal mammary tissue, precancerous lesions, and breast cancer tissue and found similar expression patterns as in TA2 SBC. Further, in order to verify the role of $\beta$-catenin in tumor growth in vivo, MA-891 cells treated with the negative control, siRNA control, and siRNA $\beta$-catenin-1512 were typsinized, resuspended, and injected into the right flank of TA2 mice. The lengths and widths of tumors were measured and the volumes were calculated to determine the growth curve (Fig.4A). All mice were sacrificed on the 19th day after initial inoculation, and the tumor tissues were collected and weighed. As shown in Figures 4B and $4 \mathrm{C}$, the average weight of xenografts in siRNA- $\beta$-catenin-1512 was significantly decreased in comparison with the negative control, and the differences between the two groups had statistical significance $(Z=-2.402, \quad P=0.018)$. These results indicated that down-regulated expression of $\beta$-catenin in TA2 breast cancer cells inhibited tumor growth. In addition, the expression level of $\beta$-catenin was found to be associated with tumor metastasis in TA2 xenografts. After TA2 mice were sacrificed, the liver and lung were examined for metastatic tumor by H\&E staining (supplementary figure $2 B$ and $2 C$ ). Some metastatic tumors were large and could be observed with the naked eye (Fig. 4D). Results of H\&E staining showed that all mice in the negative control group and the siRNA control group developed hepatic and/or lung metastases. Only one mouse had hepatic metastasis (Fig. 4E), while, two mice showed lung metastasis (Fig. 4F).
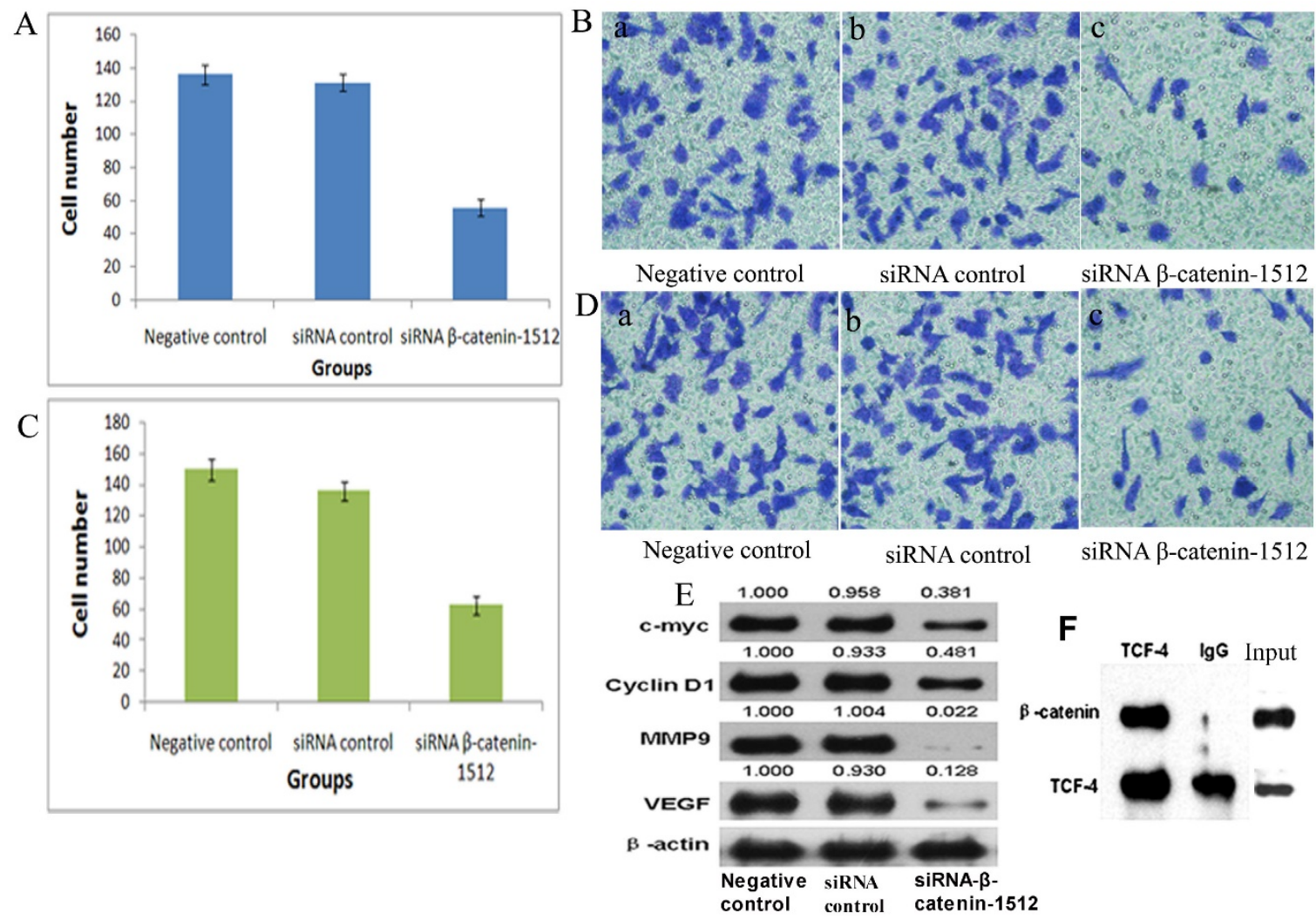

Figure 3. Low-expression of $\beta$-catenin inhibited the migration and invasion capability of MA-89 cell. A. Histogram shows the number of invading MA-891 cells with the negative control, siRNA control and siRNA $\beta$-catenin-1512 treatments. B. Results of Transwell experiments with MA-891 showing that siRNA $\beta$-catenin-1512 inhibits cell invasiveness. a) Negative control, b) siRNA control, c) siRNA $\beta$-catenin-1512. C. Histogram shows the number of migrating MA-891 cells with the negative control, siRNA control and siRNA $\beta$-catenin-1512 treatments. D. The number of migrations of MA-891 cells treated with siRNA $\beta$-catenin-1512 was lower than that found in the other two groups. a) Negative control, b) siRNA control, c) siRNA $\beta$-catenin-1512. E. The expression levels of C-myc, CyclinD1, MMP-9, and VEGF as determined by western blot analysis. F. Interaction between $\beta$-catenin and TCF-4 in MA-891 cells using Co-IP. 

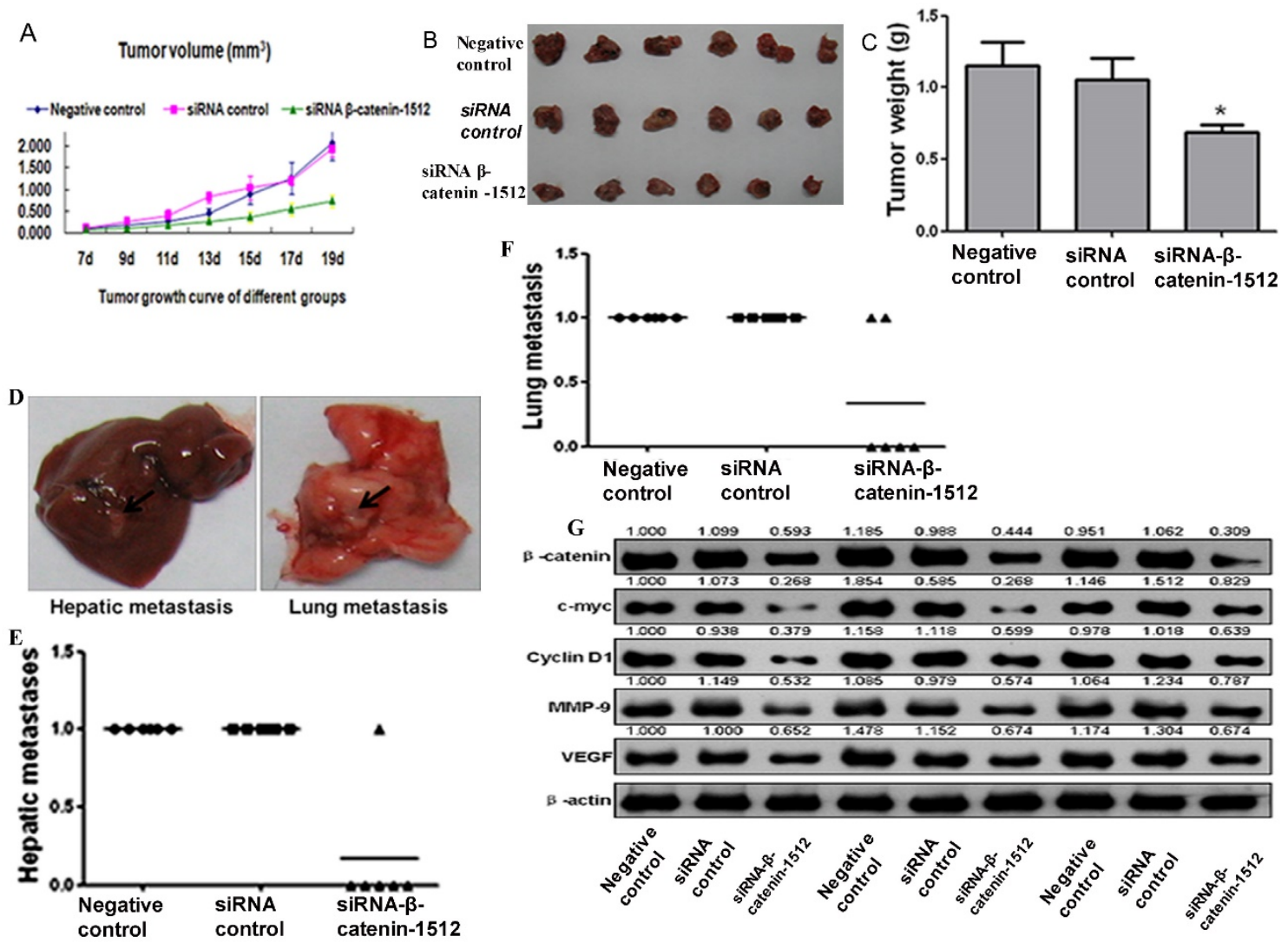

Figure 4. $\beta$-catenin activation associated with tumor growth and metastasis in cancer cells derived from TA2 SBC. A. Tumor growth curve in TA2 mice injected with MA-891 cells with negative control, siRNA control, and siRNA $\beta$-catenin-1512. The volumes of xenografts were calculated and presented as mean \pm SD. B. All the mice were sacrificed and the xenografts were removed and photographed on the 19th day after injection. C. The average tumor weight, presented as mean \pm SD. D. The gross images of hepatic and lung metastases in TA2 mice injected with MA- 891 cells with different treatments. E. Hepatic metastases were counted after H\&E staining. F. Lung metastases counted after H\&E staining. G. The expression levels of $\beta$-catenin, C-myc, CyclinD1 MMP-9, and VEGF in TA2 xenografts derived from MA-891 injection with different treatments after western blot analysis.

\section{9. $\beta$-catenin regulated levels of tumor proliferation and metastasis-related protein expression}

Proteins were extracted from tumor tissues in the three groups for western blot analysis. Compared to the negative control and siRNA control, the protein expression levels of c-myc, cyclin D1, MMP-9, and VEGF were decreased in the cells with siRNA $\beta$ catenin-1512 (Fig. 4G), indicating that $\beta$-catenin was important for tumor development and progression in TA2 SBC.

\section{Discussion}

The TA2 mouse is one of ideal animal models for breast cancer research. TA2 mice show a high incidence of SBC without any chemical stimulus. The incidence of breast cancer in TA2 mice with pregnancy reaches $84.1 \%$ and is more than TA2 virgin mice with $41.4 \%$ incidence of breast cancer. The incidence of male TA2 is only $1 \%$. Our previous studies have shown that the tumorigenesis of TA2 SBC is estradiol and progesterone-dependent. Estradiol and progesterone together induce the replication of MMTV. High titer of MMTV induces carcinogenesis in mammary epithelium [6, 20]. Results of gene expression profiles of SBC and in matched normal mammary gland tissues in TA2 mice have confirmed that the expression levels of Wnt1, Wnt5a, Wnt5b, and Wnt10b mRNAs in breast cancer tissues were higher than those in normal mammary tissues $[6,7,21]$. The detailed mechanism of tumorigenesis in TA2 mice is still unclear. In this study, thirteen tumor masses from nine TA2 mice including different stage of pathologic changes were used to investigate the expression of proteins involved in $\mathrm{Wnt} / \beta$-catenin signaling pathway. Furthermore, cell line derived from TA2 SBC was used to explore the effect of the Wnt/ $\beta$-catenin signaling pathway on tumor growth and metastasis in MA-891 xenografted breast cancer in TA2 mice. We evaluated the role of $\beta$-catenin during the proliferation and metastasis of TA2 SBC via RNAi, co-IP, and western blot.

To determine the role of $\beta$-catenin in breast cancer development in TA2 mice, we used RNAi to transfect siRNA that was targeted against $\beta$-catenin, into MA-891 cells. After comparing a panel of siRNAs, siRNA $\beta$-catenin-1512 was determined to have the 
strongest inhibitory effect and was used to study the function of $\beta$-catenin. As a key regulator in the Wnt signaling pathway, $\beta$-catenin plays an important role in the regulation of mammary development and tumorigenesis. At the plasma membrane, $\beta$-catenin aids cadherins in maintaining mammary epithelium integrity. In the nucleus, $\beta$-catenin regulates gene expression programs that are essential for mammary stem cell biology during mammary tissue development. Loss of $\beta$-catenin from cell-cell adhesive junctions or elevation in the cytosol and nucleus can occur independently by numerous routes. Importantly, both events predispose the breast to cancer $[13,14]$. Meanwhile, increased cytoplasmic and nuclear $\beta$-catenin levels are correlated with poor prognosis and patient survival [15-19].

Results of IHC staining showed that the subcellular localization of $\beta$-catenin changes during TA2 SBC development. In normal mammary epithelium, $\beta$-catenin is located in the cell membrane. However, in precancerous lesions and breast cancer tissues, $\beta$-catenin expression is located in the cytoplasm and/or nucleus. After translocating into the nucleus, $\beta$-catenin forces the transcription factors LEF1 and TCF to disengage their previous partners and drive transcription of target genes including cyclin D1 and c-myc [22-26]. The results presented here showed that there was coexpression of $\beta$-catenin and TCF-4 proteins in TA2 mice. C-myc and Cyclin $\mathrm{D} 1$ are important target genes of the $\mathrm{Wnt} / \beta$-catenin signaling pathway [13, 27-29]. Our results suggested that the expression of these genes can be regulated by $\beta$-catenin in TA2 SBC, and low expression of $\beta$-catenin in MA-891 cells by siRNA can inhibit the cell cycle and tumor growth, both in vitro and in vivo.

The rate of metastasis for TA2 SBC to the lung and/or liver is also high; the rate of metastasis to lung or liver exceeds $80 \%$ [6, 7, 21]. Synthesized and secreted by various types of malignant cells and host stromal cells, MMP-9 is a classic metastasis-promoting gene implicated in metastasis of many kinds of human cancers, including breast cancer [30-32]. Results of this study suggest that $\beta$-catenin regulates the expression levels of MMP-9 in vitro and in vivo and promotes tumor metastasis in TA2 mice associated with the expression of MMP-9. Another protein of interest that was found to be associated with the inhibition of $\beta$-catenin was VEFG. Angiogenesis is the process by which new microvessels sprout from pre-existing blood vessels. Angiogenesis is necessary for tumor growth and metastasis [33]. VEGF can stimulate the proliferation and migration of vascular endothelial cells and model the tumor neovasculature.

\section{Conclusions}

$\beta$-catenin promotes tumorigenesis and metastasis in TA2 mice and enhances the expression of downstream molecules such as c-myc, Cyclin D1, MMP-9, and VEGF. These proteins may give novel insight into our understanding of the mechanism involved in TA2 SBC. However, this study focused on the role of $\beta$-catenin in TA2 SBC development; other proteins of the Wnt signaling pathway need to be explored in the future. Furthermore, the detailed mechanisms of MMTV including its insertion site in DNA, and the induction process of epithelial proliferation in mammary gland during TA2 SBC development might provide critical clues for preventing breast cancer in individuals with MMTV homologous sequences.

\section{Supplementary Material}

Supplementary figures and tables.

http://www.jcancer.org/v08p2114s1.pdf

\section{Abbreviations}

TA2: Tientsin Albino 2; SBC: spontaneous breast cancer; int-1: integration site; wg: wingless; MMTV: mouse mammary tumor virus; RIPA: radioimmunoprecipitation assay; SDS: sodium dodecyl sulfate. FCM: flow cytometry; LEF/TCF: lymphoid enhancer factor/T-cell-specific transcription factor; MMP-9: matrix metalloproteinase 9; co-IP: co-immunoprecipitation

\section{Acknowledgments}

Grant numbers and sources of support: This work was supported in part by grants from the National Science Foundation of China (\#81472729 and \#81672426), the Foundation of Tianjin Health Bureau (\#2014KY29, \#2015KY28, and \#15KG112).

\section{Competing Interests}

The authors have no conflicts of interest.

\section{References}

1. Kaufmann M RA. Long-term risk of breast cancer recurrence: the need for extended adjuvant therapy. J Cancer Res Clin Oncol. 2005; 131: 487-94.

2. Demidov ON, Kek C, Shreeram S, Timofeev O, Fornace AJ, Appella E, et al. The role of the MKK6/p38 MAPK pathway in Wip1-dependent regulation of ErbB2-driven mammary gland tumorigenesis. Oncogene. 2007; 26: 2502-6.

3. Gregorieff A, Clevers H. Wnt signaling in the intestinal epithelium: from endoderm to cancer. Genes \& development. 2005; 19: 877-90.

4. Iorio MV, Ferracin M, Liu CG, Veronese A, Spizzo R, Sabbioni S, et al. MicroRNA gene expression deregulation in human breast cancer. Cancer research. 2005; 65: 7065-70

5. Simin K, Wu H, Lu L, Pinkel D, Albertson D, Cardiff RD, et al. pRb inactivation in mammary cells reveals common mechanisms for tumor initiation and progression in divergent epithelia. PLoS biology. 2004; 2: E22.

6. Sun B, Zhang S, Zhang D, Liu Y, Li Y, Rong Z, et al. Clusterin is associated with spontaneous breast cancer in TA2 mice. FEBS letters. 2007; 581: 3277-82.

7. Yin Y, Yang Z, Zhang S. Combined treatment with exogenous estradiol and progesterone increases the incidence of breast cancer in TA2 mice without ovaries. Cancer letters. 2011; 311: 171-6. 
8. Sallam AA, Ayoub NM, Foudah AI, Gissendanner CR, Meyer SA, El Sayed KA. Indole diterpene alkaloids as novel inhibitors of the Wnt/beta-catenin pathway in breast cancer cells. European journal of medicinal chemistry. 2013; 70C: 594-606.

9. Arend RC, Londono-Joshi AI, Straughn JM, Jr., Buchsbaum DJ. The Wnt/beta-catenin pathway in ovarian cancer: A review. Gynecologic oncology. 2013.

10. Polakis P. Wnt signaling and cancer. Genes \& development. 2000; 14: 1837-51.

11. Miller JR. The Wnts. Genome biology. 2002; 3: REVIEWS3001.

12. Moon RT, Kohn AD, De Ferrari GV, Kaykas A. WNT and beta-catenin signalling: diseases and therapies. Nature reviews Genetics. 2004; 5: 691-701.

13. Lin SY, Xia W, Wang JC, Kwong KY, Spohn B, Wen Y, et al. Beta-catenin, a novel prognostic marker for breast cancer: its roles in cyclin D1 expression and cancer progression. Proceedings of the National Academy of Sciences of the United States of America. 2000; 97: 4262-6.

14. Ozaki S, Ikeda S, Ishizaki $\mathrm{Y}$, Kurihara $\mathrm{T}$, Tokumoto $\mathrm{N}$, Iseki $\mathrm{M}$, et al. Alterations and correlations of the components in the Wnt signaling pathway and its target genes in breast cancer. Oncology reports. 2005; 14: 1437-43.

15. Karayiannakis AJ, Nakopoulou L, Gakiopoulou H, Keramopoulos A, Davaris PS, Pignatelli M. Expression patterns of beta-catenin in in situ and invasive breast cancer. European journal of surgical oncology: the journal of the European Society of Surgical Oncology and the British Association of Surgical Oncology. 2001; 27: 31-6.

16. Wong SC, Lo SF, Lee KC, Yam JW, Chan JK, Wendy Hsiao WL. Expression of frizzled-related protein and Wnt-signalling molecules in invasive human breast tumours. The Journal of pathology. 2002; 196: 145-53.

17. Prasad CP, Gupta SD, Rath G, Ralhan R. Wnt signaling pathway in invasive ductal carcinoma of the breast: relationship between beta-catenin, dishevelled and cyclin D1 expression. Oncology. 2007; 73: 112-7.

18. Prasad CP, Mirza S, Sharma G, Prashad R, DattaGupta S, Rath G, et al. Epigenetic alterations of $\mathrm{CDH} 1$ and APC genes: relationship with activation of Wnt/beta-catenin pathway in invasive ductal carcinoma of breast. Life sciences. 2008; 83: 318-25.

19. Harborth J, Elbashir SM, Bechert K, Tuschl T, Weber K. Identification of essential genes in cultured mammalian cells using small interfering RNAs. Journal of cell science. 2001; 114: 4557-65.

20. Sun B, Zhang S, Zhang D, Li Y, Zhao X, Luo Y, et al. Identification of metastasis-related proteins and their clinical relevance to triple-negative human breast cancer. Clinical cancer research: an official journal of the American Association for Cancer Research. 2008; 14: 7050-9.

21. Gu Y, Zhang S, Wu Q, Xu S, Cui Y, Yang Z, et al. Differential expression of decorin, EGFR and cyclin D1 during mammary gland carcinogenesis in TA2 mice with spontaneous breast cancer. Journal of experimental \& clinical cancer research: CR. 2010; 29: 6.

22. Saldanha G, Ghura V, Potter L, Fletcher A. Nuclear beta-catenin in basal cell carcinoma correlates with increased proliferation. The British journal of dermatology. 2004; 151: 157-64.

23. Zhang J, Gill AJ, Issacs JD, Atmore B, Johns A, Delbridge LW, et al. The Wnt/beta-catenin pathway drives increased cyclin D1 levels in lymph node metastasis in papillary thyroid cancer. Human pathology. 2012; 43: 1044-50.

24. Poy F, Lepourcelet M, Shivdasani RA, Eck MJ. Structure of a human Tcf4-beta-catenin complex. Nature structural biology. 2001; 8: 1053-7.

25. Graham TA, Ferkey DM, Mao F, Kimelman D, Xu W. Tcf4 can specifically recognize beta-catenin using alternative conformations. Nature structural biology. 2001; 8: 1048-52.

26. Sampietro J, Dahlberg CL, Cho US, Hinds TR, Kimelman D, Xu W. Crystal structure of a beta-catenin/BCL9/Tcf4 complex. Molecular cell. 2006; 24: 293-300.

27. Gillett C, Fantl V, Smith R, Fisher C, Bartek J, Dickson C, et al. Amplification and overexpression of cyclin D1 in breast cancer detected by immunohistochemical staining. Cancer research. 1994; 54: 1812-7.

28. Aulmann S, Bentz M, Sinn HP. C-myc oncogene amplification in ductal carcinoma in situ of the breast. Breast cancer research and treatment. 2002; 74: 25-31.

29. Bieche I, Laurendeau I, Tozlu S, Olivi M, Vidaud D, Lidereau R, et al Quantitation of MYC gene expression in sporadic breast tumors with a real-time reverse transcription-PCR assay. Cancer research. 1999; 59: 2759-65.

30. Coussens LM, Werb Z. Matrix metalloproteinases and the development of cancer. Chemistry \& biology. 1996; 3: 895-904.

31. Deryugina EI, Quigley JP. Matrix metalloproteinases and tumor metastasis. Cancer metastasis reviews. 2006; 25: 9-34.

32. Stamenkovic I. Matrix metalloproteinases in tumor invasion and metastasis. Seminars in cancer biology. 2000; 10: 415-33.

33. Paku S, Kopper L, Nagy P. Development of the vasculature in "pushing-type" liver metastases of an experimental colorectal cancer. International journal of cancer Journal international du cancer. 2005; 115: 893-902. 